\title{
USP15 inhibits multiple myeloma cell apoptosis through activating a feedback loop with the transcription factor NF-kBp65
}

\author{
Lili Zhou ${ }^{1,2}$, Hua Jiang ${ }^{2}$, Juan $\mathrm{Du}^{2}$, Lu Li', Rong $\mathrm{Li}^{2}$, Jing $\mathrm{Lu}^{2}$, Weijun $\mathrm{Fu}^{2}$ and Jian $\mathrm{Hou}^{3}$
}

\begin{abstract}
USP15 has been shown to stabilize transcription factors, to be amplified in many cancers and to mediate cancer cell survival. However, the underlying mechanism by which USP15 regulates multiple myeloma (MM) cell proliferation and apoptosis has not been established. Here, our results showed that USP15 mRNA expression was upregulated in MM patients. USP15 silencing induced MM cell proliferation inhibition, apoptosis, and the expression of nuclear and cytoplasmic NF-kBp65, while USP15 overexpression exhibited an inverse effect. Moreover, in vivo experiments indicated that USP15 silencing inhibited MM tumor growth and NF-KBp65 expression. PDTC treatment significantly inhibited USP15 overexpression-induced cell proliferation, apoptosis inhibition, and NF-kBp65 expression. USP15 overexpression promoted NF-kBp65 expression through inhibition of its ubiquitination, whereas NF-kBp65 promoted USP15 expression as a positive regulator. Taken together, the USP15-NF-KBp65 loop is involved in MM tumorigenesis and may be a potential therapeutic target for MM.
\end{abstract}

\section{Introduction}

Multiple myeloma (MM) is a malignant blood system disease derived from B cells. It is characterized by clonal expansion of plasma cells in the bone marrow, which secretes a large number of monoclonal immunoglobulins, together with a series of dissolved bone lesions, clinical symptoms, and organ dysfunction, such as bone disease, pathological fractures, renal failure, and anemia ${ }^{1,2}$. MM constitutes approximately $1 \%$ of all malignant tumors and is the second most common blood system tumors, surpassed only by lymphoma ${ }^{3}$. The MM mortality is as high as $70-90 \%$. Since the pathogenesis of MM is complex, the number and structural abnormalities of chromosomes, activation of oncogenes, inactivation of tumor suppressors, IL-6-dependent cytokine network disorders, and

Correspondence: Jian Hou (houjian@medmail.com.cn)

or Weijun Fu (fuweijun@smmu.edu.cn)

${ }^{1}$ Shanghai Jiahui International Hospital Cancer Center, Shanghai 200233, China

${ }^{2}$ Department of Hematology, Shanghai Changzheng Hospital, Second Military Medical University, Shanghai 200433, China

Full list of author information is available at the end of the article. changes in bone marrow microenvironment are all related to the occurrence of myeloma ${ }^{4,5}$. With the application of proteasome inhibitors and immunomodulators, the therapeutic efforts in MM patients have improved ${ }^{6}$. The 5 and 10-year survival rates of patients with MM were increased from 32.8 and $15 \%$ to 40.3 and $20.8 \%$, respectively ${ }^{7}$. However, because of many problems such as multidrug resistance and associated side effects, $M M$ is still an incurable hematologic tumor. Therefore, it is important to further study the molecular mechanism and find more potential therapeutic targets for the treatment of MM.

Ubiquitination is a post-translational protein modification process that connects single or multiple ubiquitin molecules to a target protein and affects its stability and function. Deregulation of the deubiquitination process is frequently associated with tumorigenesis ${ }^{8,9}$. Ubiquitin-specific proteases (USPs) are deubiquitinating enzymes that reverse the ubiquitination through removing ubiquitin from the targeted proteins by directly interacting with substrates or indirectly binding to an adaptor protein such as E3 ubiquitin ligase. USP15 functions with the E3 ubiquitin ligase TRIM25 
to positively regulate type I interferon responses and to promote pathogenesis during neuroinflammation ${ }^{10}$. USP15 also regulates certain mutant versions of $\mathrm{p} 53$ and binds to and stabilizes p53 through deubiquitination in osteosarcoma and ovarian cancer cells ${ }^{11,12}$. Reduced accumulation of IkB$\alpha$ after its TNF- $\alpha$-induced degradation was observed in HeLa cells with suppression of USP15 expression, suggesting nuclear translocation of NF- $\mathrm{B}$ in TNF- $\alpha$-stimulated cells $^{13}$. Additionally, USP15 silencing also abolished the inhibitory effect of morphine on NF- $\mathrm{KB}$ signaling ${ }^{14}$. However, the correlation between USP15 and NF- $\mathrm{kB}$ and the effect of USP15 on apoptosis in MM are still unclear.

The highly abnormal and persistently activated NF- $\mathrm{kB}$ is associated with the proliferation, cell cycle process, apoptosis, metabolism, and drug resistance of $\mathrm{MM}^{15,16}$. The ubiquitination process is involved in the activation of the NF- $\mathrm{KB}$ pathway through degradation of I $\mathrm{I} B-\alpha$ and activation of IкB kinase. Regulation of the ubiquitination process therefore directly affects the activation of NF- $\mathrm{KB}^{17}$. In this study, we have evaluated the biological functions of USP15 in apoptosis and proliferation of MM cells and the underlying molecular mechanisms involved. Upregulation of USP15 was in MM patients was found to induce cell proliferation and inhibit cell apoptosis of MM through activating NF-kB signaling. USP15 promoted NF-кBp65 expression through inhibiting ubiquitination. USP15 inhibited MM cell apoptosis through activating a feedback loop with NF-kBp65.

\section{Materials and methods Clinical samples}

Ninety-five cases of bone marrow samples from 80 patients with $\mathrm{MM}$ and 15 patients with proliferative bone marrow (PBM) were collected in Changzheng Hospital from March 2011 to May 2017. Written informed consent was obtained from all participants in this study. The study protocol was approved by the ethics committee of Changzheng Hospital.

\section{Cell culture}

RPMI 8226, U266, H929, KMS12, and KMS18 human MM cell lines obtained from the Cell Bank of the Chinese Academy of Science (Shanghai, China) and non-cancerous bone marrow-derived plasma cells (control) were cultured in RPMI-1640 medium (Hyclone, USA) containing 10\% fetal bovine serum (GIBCO) and 1\% antibiotic (mixtures of penicillin and streptomycin, Solarbio) in a $37^{\circ} \mathrm{C}, 5 \% \mathrm{CO}_{2}$ incubator (Thermo, USA). The old medium was replaced with fresh medium depending on the growth of the cells during the period of culture.

\section{Cell transfection}

Two siRNAs targeting human USP15 (point 1, 10771095, 5'-GAGGTGAAATAGCTAAATC-3'; point 2,
1754-1772, 5'-GATACAGAGCACGTGATTA-3') were produced and transfected into the RPMI 8226 and U266 cells using Lipofectamine 2000 (Invitrogen, USA) following the manufacturer's protocol. The coding sequence of USP15 was synthesized using the primers containing the restriction enzyme cut sites of BamHI and EcoRI (USP15 Forward: 5'-GCGAATTCATGGCGGAAGGCG GAGCGGCGGAT-3' and Reverse: 5'-CGGGATCCT TAGTTAGTGTGCATACAGTTTTC-3') and integrated into the pLVX-Puro plasmid to increase USP15 expression. Recombinant plasmids along with the psPAX2 and pMD2G packaging plasmids were co-transfected into 293 $\mathrm{T}$ cells using Lipofectamine 2000 (Invitrogen, USA). Forty-eight $\mathrm{h}$ after transfection, recombined lentiviral vectors were collected and used to infect RPMI 8226 and U266 cells. Cells with scrambled siRNA (siNC) and blank pLVX-Puro (Vector) transfection were used as negative control.

\section{CCK-8 assay}

The Cell Counting Kit (CCK)-8 (Beyotime, Shanghai, China) was used to examine $\mathrm{MM}$ cell proliferation. Briefly, cells $\left(5 \times 10^{3}\right.$ cell/well $)$ cultured in complete medium containing the NF- $\mathrm{kB}$ inhibitor PDTC $(50 \mu \mathrm{M})$ or no inhibitor were processed following the standard procedure in 96-well plates and were maintained in 5\% $\mathrm{CO}_{2}$ incubator at $37^{\circ} \mathrm{C}$ overnight. After $0,24,48$ and $72 \mathrm{~h}$ transfections of RPMI 8226 and U266 cells with siUSP15\#1, siUSP15\#2 or pLVX-Puro-USP15, the CCK-8 solution $(10 \mu \mathrm{l}$ per well) was added to the cells which were then maintained in a $\mathrm{CO}_{2}$ incubator for $1 \mathrm{~h}$ at $37^{\circ} \mathrm{C}$, after which the absorbance readings were obtained at $450 \mathrm{~nm}$.

\section{Cell apoptosis assay}

In accordance with the manufacturer's instructions, the apoptosis of MM cells was analyzed using the Annexin VFITC/Propidium Iodide (PI) cell apoptosis kit (BD Biosciences, USA). RPMI 8226 and U266 cells cultured in complete medium containing PDTC $(50 \mu \mathrm{M})$ or no inhibitor were transfected with siUSP15\#1, siUSP15\#2 or pLVX-Puro-USP15 for $48 \mathrm{~h}$, washed three times with PBS, digested by trypsin, centrifuged (1000xg, room temperature) for $10 \mathrm{~min}$, adjusted to $5 \times 10^{5} \mathrm{cell} / \mathrm{ml}$ and suspended in the Annexin V-FITC and PI-binding buffer. The apoptotic cells were measured with flow cytometry (BD Biosciences) after $10 \mathrm{~min}$ of incubation in the dark.

\section{Luciferase reporter experiments}

RPMI 8226 and U266 cells $\left(5 \times 10^{5}\right.$ cell/well) cultured in complete medium containing PDTC $(50 \mu \mathrm{M})$ or the NF$\kappa B$-agonist LPS $(1 \mu \mathrm{g} / \mathrm{ml})$ were seeded in a 6-well plate, cultured in an incubator with $5 \% \mathrm{CO}_{2}$ at $37^{\circ} \mathrm{C}$ for $24 \mathrm{~h}$, and then transfected with $1.5 \mu \mathrm{g}$ of the pGL3-basic plasmid containing the USP 15 promoter at $37^{\circ} \mathrm{C}$ for $6 \mathrm{~h}$ using 
Lipofectamine 2000 (Invitrogen) following the manufacturer's protocol. Forty-eight $\mathrm{h}$ after transfection, $100 \mu \mathrm{l}$ of the luciferase assay reagent and $10 \mu \mathrm{l}$ of the Stop\&Glo reagent were added into the RPMI 8226 and U266 cells. Luciferase activity (Firefly and Renilla) was measured with the Dual-Luciferase Reporter assay system (Promega) according to the manufacturer's protocol.

\section{Real-time PCR}

We applied Real-time PCR to detect the mRNA levels of USP15, Caspase-3, PARP1, Bcl-2, Bcl-xL and Survivin. First, extraction of total RNA from MM tissues and cell lines was achieved by TRIzol (Invitrogen). Subsequently, the TaqMan reverse transcription kit (Applied Biosystems, USA) was used to reverse the isolated RNA into cDNA. Real-time PCR was performed using the SYBR Green qRT-PCR kit (Promega, USA) on an ABI7500 system following the manufacturer's instructions to analyze the mRNA levels normalizing to GAPDH. The following are the primer sequences: USP15, 5'-TGCCT ACTTCCAACTCTC-3' and 5'-GCTCTTCCTTTCCTT CTC-3'; Caspase-3, 5'-TGGTTCATCCAGTCGCTTTG$3^{\prime}$ and $5^{\prime}$-AAATTCTGTTGCCACCTTTCG-3'; PARP1, 5'-TCACGGACACGCTTTCACC-3' and 5'-CCCCGCA GATTCTACATTCG-3'; Bcl-2, 5'-GCAGTGTGGTCTC CGAATGTC- $3^{\prime}$ and $5^{\prime}$-CATTGCCTCTCCTCACGTTC C-3'; Bcl-xL, 5'-CAGGTATGGAAGGGTTTG-3' and 5'TAGGGATGGAAGGAAAGG-3'; Survivin, 5' ${ }^{\prime}$-CCACCG CATCTCTACATTC-3' and 5'-CTTTCTCCGCAGTT TCCTC-3'; GAPDH, 5'-AATCCCATCACCATCTTC-3' and $5^{\prime}$-AGGCTGTTGTCATACTTC-3'. The relative expression of mRNAs was calculated through the $2^{-\Delta \Delta C T}$ method.

\section{Western blotting}

Total protein was extracted using a total protein extraction buffer (Beyotime, China). A 10\% sodium dodecyl sulfate polyacrylamide gel was prepared to isolate the proteins. After transferred to a nitrocellulose membrane, the protein bands were blocked with $5 \%$ non-fat milk. The blots were then incubated with primary antibodies and secondary antibodies. The antibodies and reagents used were as follows: USP15 (Abcam, ab71713, 1:1000); active Caspase-3 (Abcam, ab2302, 1:200); cleaved PARP1 (Abcam, ab32064, 1:1000); Survivin (Abcam, ab76424, 1:3000); Bcl-2 (Abcam, ab32124, 1:1000); Bcl-xL (Abcam, ab32370, 1:1000); NF-кBp65 (Cell Signaling Technology, \#8242, 1:1000); IкB- $\alpha$ (Cell Signaling Technology, \#9242, 1:1000); p-ІкB- $\alpha$ (Cell Signaling Technology, \#2859, 1:1000); H3 (Cell Signaling Technology, \#4499S, 1:2000); GAPDH (Cell Signaling Technology, \#5174, 1:2000); HRP-labeled Goat Anti-Rabbit IgG (Beyotime, A0208, 1:1000); HRP-labeled Donkey AntiGoat IgG (Beyotime, A0181, 1:1000); and HRP-labeled
Goat Anti-Mouse IgG (Beyotime, A0216, 1:1000). Signals were detected using an enhanced chemiluminescence Western Blotting substrate (Pierce; Thermo Fisher Scientific, Inc., USA).

\section{Co-immunoprecipitation (Co-IP) and ubiquitination in vitro}

Co-IP was performed as previously described ${ }^{18}$. Briefly, cold PBS was used to wash the cells three times, and the cells were scraped into lysis buffer containing complete protease inhibitors and centrifuged at $14,000 \times g$ for $20 \mathrm{~min}$ at $4{ }^{\circ} \mathrm{C}$. The supernatants were incubated with anti-NFкBp65 (1:1000), anti-IкB- $\alpha$ (1:1000) or normal IgG (1:1000) antibody, and the immunocomplexes were then associated with protein A-sepharose. Anti-NF- $\mathrm{kBp} 65$ (1:1000), anti-IKB- $\alpha$ (1:1000) and anti-ubiquitin (1:2000) antibodies were used for western blot analysis.

\section{Animal experiments}

For the tumor growth assays in vivo, RPMI 8226 cells with pLKO.1-USP15-shRNA or negative control (shNC) transfection were resuspended in PBS at a concentration of $1 \times 10^{7}$ cells $/ \mathrm{ml}$. The cell suspension $(100 \mu \mathrm{l})$ was injected into the right armpit of BALB/c male nude mice (4-5 week-old; 6 per group). Forty-eight days after injection, the mice were sacrificed, and tumor tissues were excised and weighed. Animal experiments were performed according to the legal requirements and were approved by the Changzheng Hospital institutional ethical committee.

\section{Statistical analysis}

Data are presented as the mean $\pm \mathrm{SD}$, and each test was repeated at least three times. Statistical analysis was conducted using one-way ANOVA with GraphPad Prism software, version 5 (GraphPad Software, USA). $P<0.05$ was regarded as statistically significant.

\section{Results \\ USP15 mRNA expression in MM tissues and cell lines}

The mRNA expression of USP15 in the bone marrow of patients with MM $(n=80)$ was upregulated compared to patients with PBM; $(n=15)$ (Fig. 1a). Moreover, the mRNA expression of USP15 in five different MM cell lines and in control cells, which were non-cancerous bone marrowderived plasma cells, was also measured. We found that USP15 expression was higher in MM cell lines compared with control cells, with the highest expression detected in RPMI 8226 and U266 cells compared with other MM cell lines (Fig. 1b, c). These results suggest that USP15 may participant in the development and progression of MM.

\section{USP15 overexpression promotes MM cell proliferation}

To evaluate the role of USP15 in MM tumorigenesis in vitro, RPMI 8226 and U266 cells were transfected with 

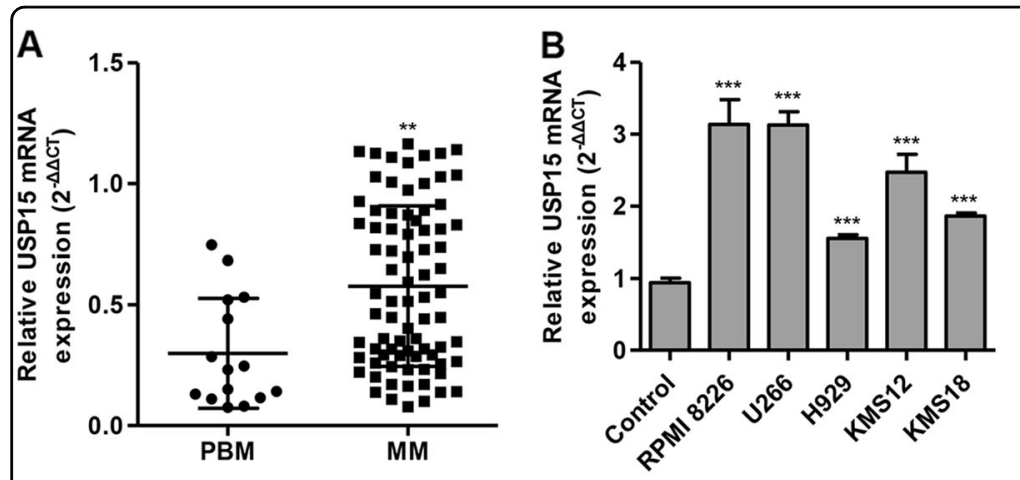

C

Fig. 1 USP15 expression in MM tissues and cell lines. a The mRNA expression of USP15 in bone marrow samples from 80 patients with multiple myeloma (MM) and 15 patients with proliferative bone marrow (PBM) was measured by Real-time PCR. The expression of USP15 in five different MM cell lines and in control cells, non-cancerous bone marrow-derived plasma cells, was measured by Real-time PCR (b) and western blotting (c). ${ }^{* *} P<0.01$ compared with PBM or control

siUSP15\#1, siUSP15\#2, or pLVX-Puro-USP15. As shown in Fig. 2a, siUSP15\#1 and siUSP15\#2 transfection in RPMI 8226 cells significantly reduced USP15 protein expression by $61.7 \%$ and $66.9 \%$ compared to siNC, respectively. siUSP15\#1 and siUSP15\#2 transfection in U266 cells significantly reduced USP 15 protein expression by $55.2 \%$ and $72.2 \%$ compared to siNC, respectively (Fig. 2c). pLVX-Puro-USP15 transfection in RPMI 8226 and U266 cells significantly increased USP 15 protein expression by 1.79-fold and 1.28-fold compared to the blank vector, respectively (Fig. 2b, d).

Furthermore, the CCK- 8 assay demonstrated that siUSP15\#1 and siUSP15\#2 transfection in RPMI 8226 cells significantly inhibited cell proliferation by $30.3 \%$ and $37.2 \%$, respectively, at $48 \mathrm{~h}$ after transfection compared to the vector plus siNC group (Fig. 2e). siUSP15\#1 and siUSP15\#2 transfection in U266 cells significantly inhibited cell proliferation by $31.0 \%$ and $38.2 \%$, respectively, at $48 \mathrm{~h}$ after transfection compared to the vector plus siNC group (Fig. 2f). pLVX-PuroUSP15 transfection in RPMI 8226 and U266 cells significantly increased cell proliferation by $35.6 \%$ and $36.5 \%$, respectively, at $48 \mathrm{~h}$ after transfection compared to the vector plus siNC group (Fig. 2e, f). These data indicate that USP15 has a pro-proliferative effect in MM cells.

\section{USP15 overexpression inhibits MM cell apoptosis}

Flow cytometry analysis was performed to examine the effect of USP15 on MM cell apoptosis. We found that siUSP15\#1 and siUSP15\#2 transfection in RPMI 8226 and U266 cells significantly promoted cell apoptosis compared to the vector plus siNC group (Fig. 3a, b). pLVX-Puro-USP15 transfection in RPMI 8226 and U266 cells significantly inhibited cell apoptosis compared to the vector plus siNC group. These results suggest that USP15 has an anti-apoptotic effect in MM cells.

\section{USP15 overexpression promotes the expression of Bcl-2,} $\mathrm{BCl}-\mathrm{xL}$, Survivin, and NF-KBp65

To investigate the molecular mechanism of USP15 in the regulation of proliferation and apoptosis in MM cells, the expression of Caspase-3, PARP1, Bcl-2, Bcl-xL, Survivin, nuclear NF-кBp65, and cytoplasmic NF-кBp65 was measured by real-time PCR and/or western blot. As shown in Fig. 4a, b, siUSP15\#1 and siUSP15\#2 transfection in RPMI 8226 and U266 cells significantly reduced the mRNA expression of Bcl-2, Bcl-xL and Survivin but induced Caspase-3 and PARP1 mRNA expression compared to the vector plus siNC group. Further, transfection with siUSP15\#1 and siUSP15\#2 also mediated the expression of these proteins and inhibited nuclear NFкBp65 and cytoplasmic NF-кBp65 expression (Fig. 4c-g). However, pLVX-Puro-USP15 transfection had an inverse effect on expression of these proteins. Given the effects of USP15 on NF-kBp65 expression, the role of USP15 in the expression and ubiquitination of IкB- $\alpha$ was also measured. As shown in Fig. 4h and I, USP15 overexpression and silencing had no effect on the expression of IкB- $\alpha$ and p-IKB- $\alpha$ in U266 cells, and its overexpression did not affect IKB- $\alpha$ ubiquitination, which suggests that USP15 may regulate NF-kBp65 expression in an IKB- $\alpha$ independent manner. Additionally, USP15 overexpression significantly inhibited the ubiquitination of NF-kBp65 (Fig. 4j). These results suggest that USP15 promotes NF-kBp65 expression through deubiquitination.

\section{USP15 knockdown inhibits MM tumor growth and protein expression in vivo}

To evaluate the function of USP15 in MM in vivo, RPMI 8226 cells transfected with pLKO.1-USP15-shRNA or shNC 

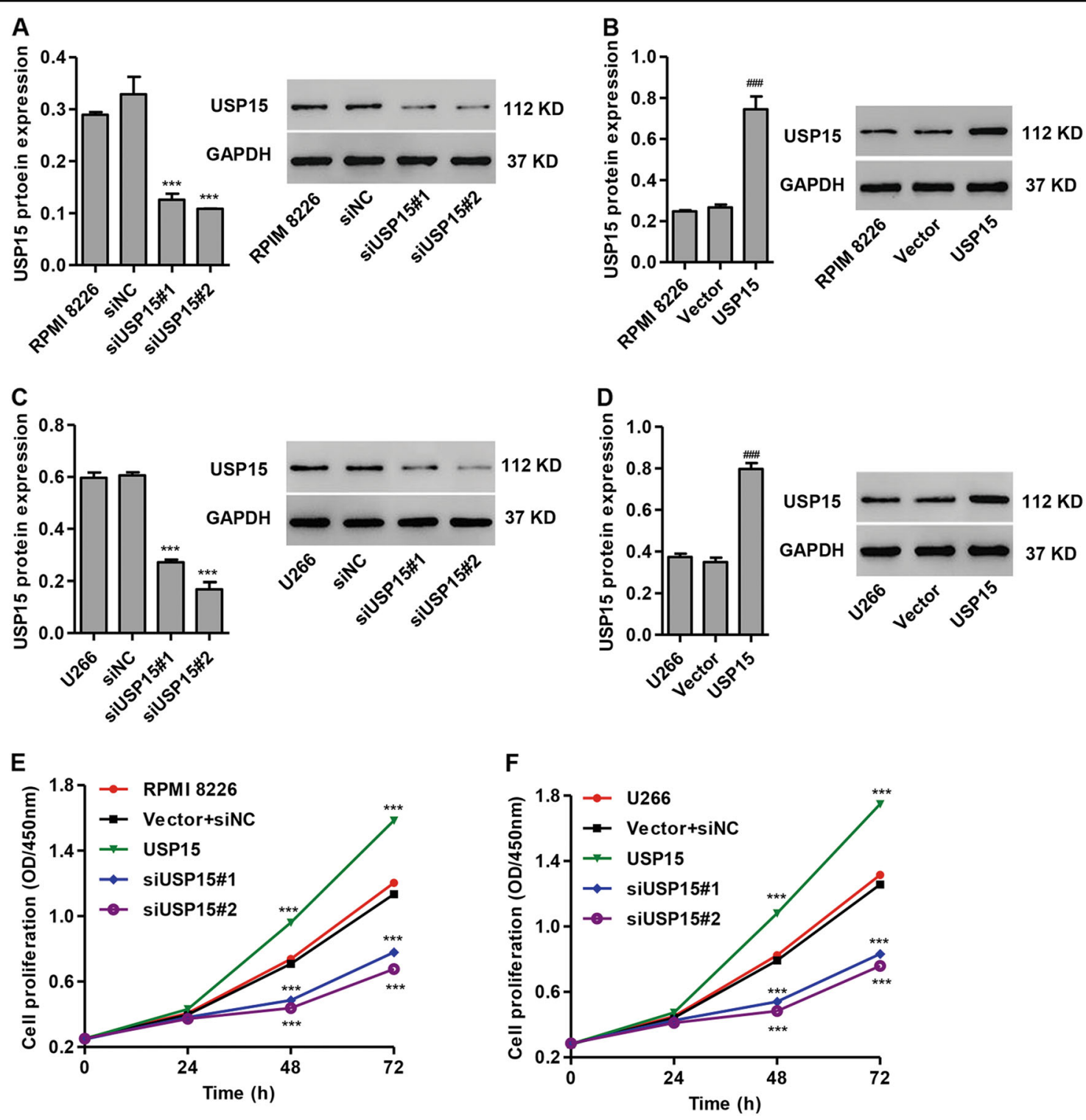

Fig. 2 Effects of USP15 RNA-interference and USP15 overexpression on MM cell proliferation. After RPMI 8226 and U266 cells were transfected with siNC plus empty pLVX-Puro (vector), siUSP15\#1, siUSP15\#2, or pLVX-Puro-USP15, the expression of USP15 was measured by western blot (a-d) and cell proliferation was measured by the CCK-8 assay $(\mathbf{e}, \mathbf{f}) .{ }^{* * *} P<0.001$ compared with siNC, vector or vector $+\operatorname{siNC}$

were injected into nude mice. We found that mice with pLKO.1-USP15-shRNA injection exhibited decreased tumor weight and tumor volume (Fig. $5 \mathrm{a}-\mathrm{c})$. The expression of USP15, Bcl-2, Bcl-xL, Survivin, nuclear NF-kBp65, and cytoplasmic NF-kBp65 was significantly decreased, but the expression of cleaved Caspase-3 and PARP1 was increased in xenograft tumors with pLKO.1-USP15-shRNA injection compared to the shNC injection (Figs. 5d, e).

\section{PDTC treatment inhibits USP15 overexpression-induced MM cell proliferation and apoptosis inhibition}

To investigate the role of NF- $\mathrm{kBp} 65$ signaling in USP15mediated MM cell proliferation and apoptosis, the NF-
кBp65 signaling inhibitor PDTC was added to MM cell cultures. As shown in Fig. 6a, b, PDTC treatment significantly inhibited the USP15 overexpression-induced proliferation of RPMI 8226 and U266 cells by 24.9 and $29.2 \%$ at $48 \mathrm{~h}$ after transfection, respectively. PDTC treatment also significantly inhibited the USP15 overexpression-induced decrease in apoptosis of RPMI 8226 and U266 cells by 7.9-fold and 5.3-fold, respectively (Fig. 6c, d). Moreover, the expression of Bcl-2, Bcl-xL, Survivin, nuclear NF-кBp65, and cytoplasmic NF-кBp65 was significantly decreased, and the expression of cleaved Caspase-3 and PARP1 was increased in MM cells after PDTC treatment (Fig. 7). These results suggest that 


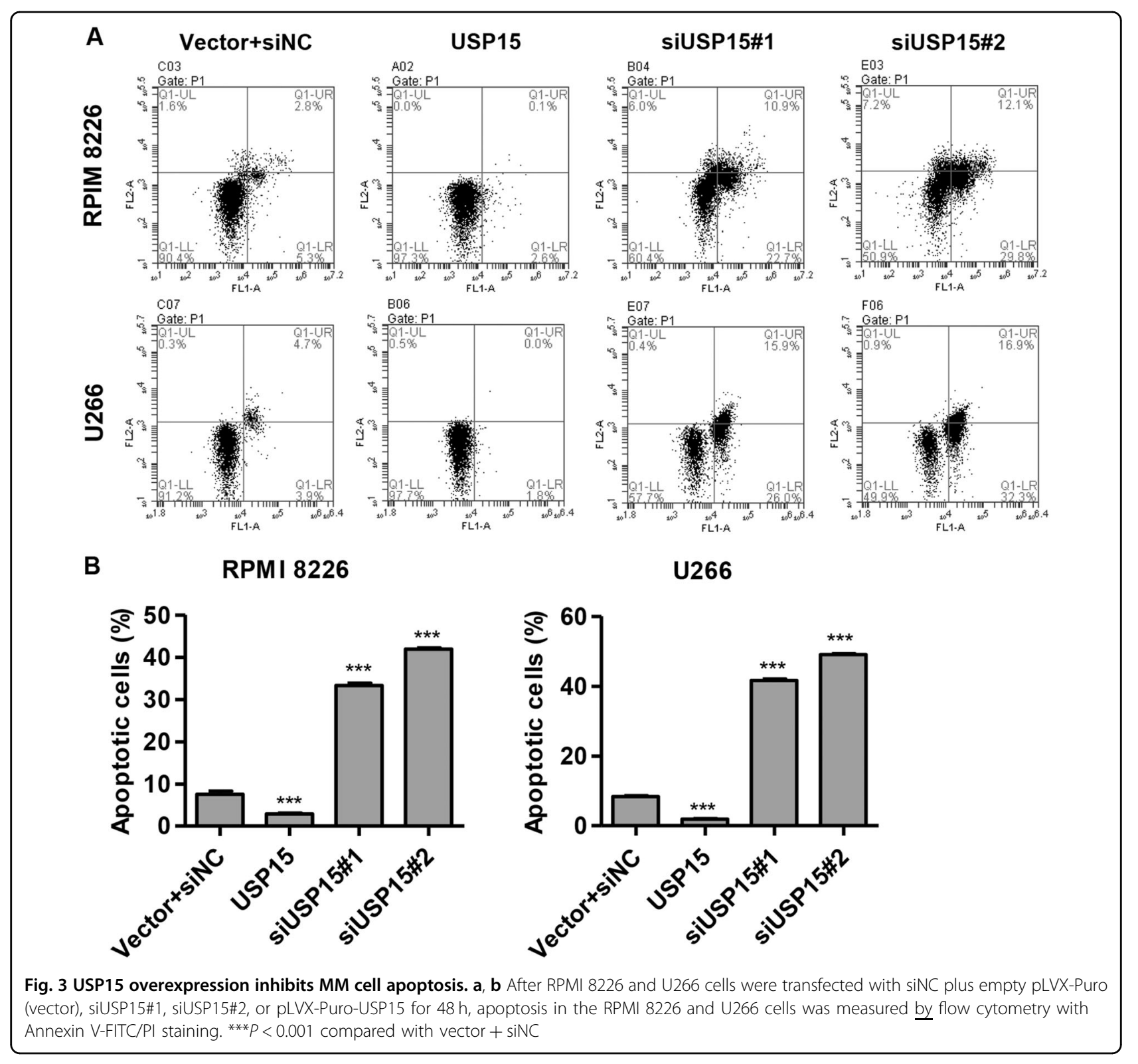

USP15 regulates MM cell proliferation and apoptosis through NF-кBp65 signaling.

\section{Evidence of an NF-KBp65-USP15 regulatory loop in MM cells}

Given the role of NF-kBp65 in transcriptional regulation, we evaluated the possible effect of NF-kBp65 on the regulation of USP15 mRNA by transfecting MM cells with a luciferase reporter vector containing the $3^{\prime} \mathrm{UTR}$ region of USP15 with or without PDTC $(50 \mu \mathrm{M})$ or LPS $(1 \mu \mathrm{g} / \mathrm{ml})$ treatment. As shown in Fig. 8a, b, PDTC treatment significantly inhibited the expression of nuclear NF-кBp65 and cytoplasmic NF- $\mathrm{kBp} 65$, but LPS treatment promoted the expression of NF-kBp65 in RPMI 8226 and U266 cells.
Whereas PDTC treatment significantly decreased the luciferase Firefly/Renilla ratio, LPS treatment significantly increased that ratio when compared to untreated cells, suggesting that NF-kBp65 enhances the promoter activity of USP15 (Fig. 8c, d). In addition, PDTC treatment in RPMI 8226 and U266 cells also significantly decreased USP15 mRNA and protein expression, but LPS treatment significantly increased their expression compared to untreated cells (Fig. 8e-h). These data indicate that the NF-kBp65-USP15 regulatory loop exists in MM cells.

\section{Discussion}

Multiple myeloma (MM) is a malignant hematological tumor with monoclonal plasma cell hyperplasia, and it 


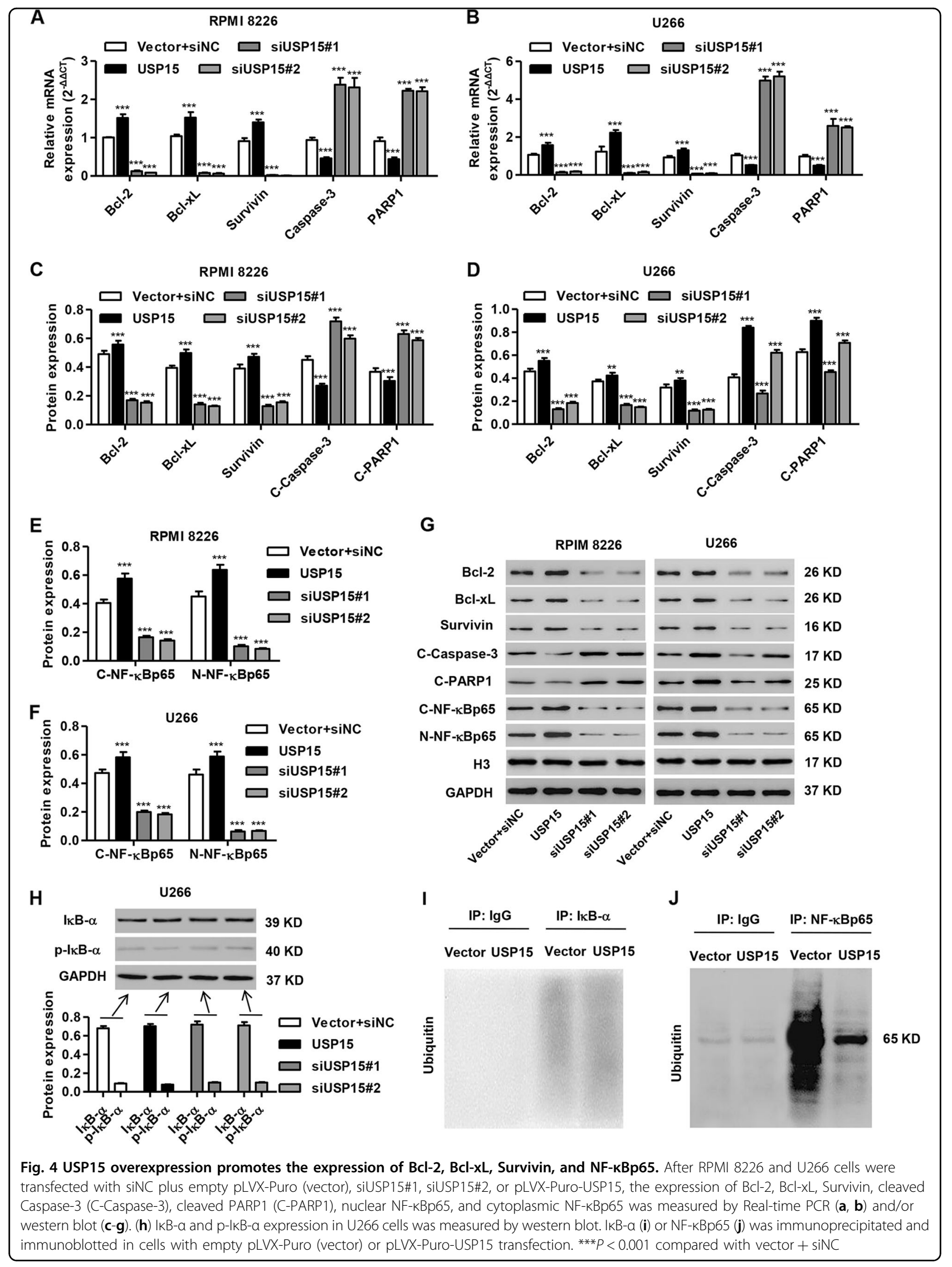




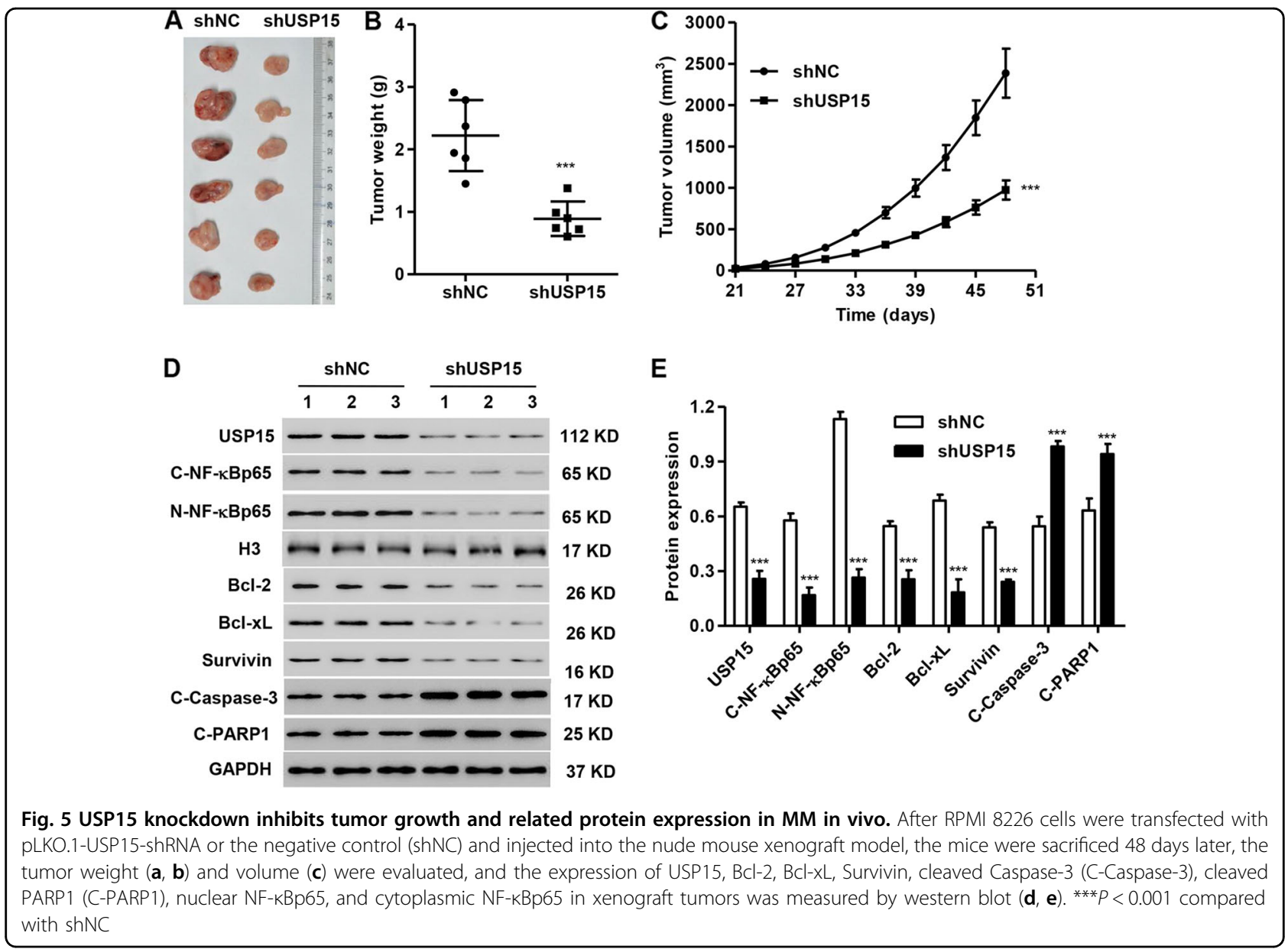

accounts for $10-15 \%$ of all blood system tumors. At present, combined chemotherapy is the main treatment for MM. Despite the continuous improvements in chemotherapy, this treatment does not cure the disease because many patients are unable to tolerate or resistant to conventional chemotherapy, and therefore experience a relapse or even death after a few months or several years ${ }^{19,20}$. In the present study, we found that USP15 mRNA expression was upregulated in the bone marrow of MM patients compared to patients with -PBM. Silencing USP15 inhibited MM cell growth both in vitro and in vivo. Overexpression of USP15 promoted proliferation and inhibited apoptosis, and these effects were inhibited by PDTC treatment. NF-kBp65 positively regulated USP15 expression, whereas USP15 overexpression promoted NF- $\mathrm{KBp} 65$ expression through deubiquitination of NF-kBp65 in MM cells. The USP15NF-kBp65 regulatory loop was involved in MM cell apoptosis (Fig. 8i).

Increasing evidence has demonstrated that alterations in the deubiquitinating enzymes, including the ubiquitinspecific peptidase (USP) families, are implicated in the pathogenesis of a wide variety of tumors. A previous study showed that USP4 is upregulated in melanoma tissues and that it accentuates the migration and invasion of melanoma cells by promoting the epithelialmesenchymal transition but has no effect on cell proliferation $^{21}$. USP21 is upregulated in renal cell carcinoma tissues and cell lines, and depletion of USP21 inhibits cell proliferation and invasion through binding to the IL-8 promoter region and mediating transcriptional initiation $^{22}$. USP14 is upregulated in oral squamous cell carcinoma tissues and cell lines; reducing USP14 levels inhibits cell viability and tumor growth in vivo and induces cell apoptosis in vivo but not in vitro ${ }^{23}$. These findings suggest that USPs may act as oncogenes associated with cancer tumorigenesis. In the present study, our results showed that USP15 is upregulated in MM patients and has pro-proliferative and anti-apoptotic roles in MM cells and xenograft tumors in nude mice, which is similar to the role in promoting oncogenesis observed in other cancers others ${ }^{24,25}$.

To investigate the molecular mechanism by which USP15 regulates MM cell proliferation and apoptosis, 


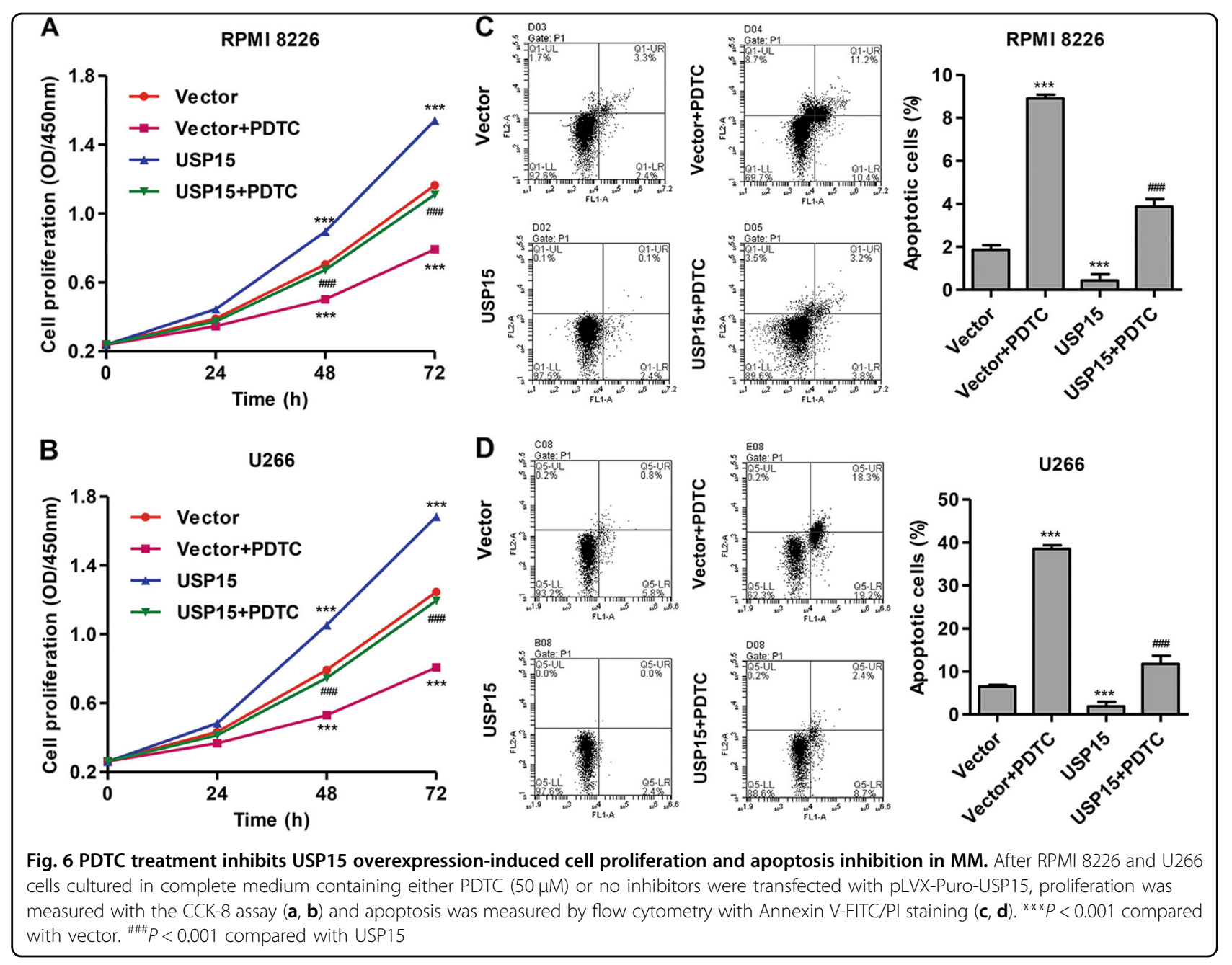

NF-kBp65 expression was also measured. NF-кBp65 expression was positively regulated by USP 15 through inhibition of its ubiquitination in MM cells. The PDTC treatment-induced inhibition of NF-kBp65 in MM cells inhibited the USP15-induced reduction in apoptosis. Conversely, NF-kBp65 also enhanced the promoter activity of USP15 and its protein expression. Studies focused on the other USPs demonstrated that USP14 overexpression regulates IкB polyubiquitination to stimulate its degradation ${ }^{26}$, and ectopic expression of USP4 inhibits the TRAF2- and TRAF6-stimulated NF$\kappa B$ reporter gene and negatively regulates the TNF- $\alpha-$ induced I $\kappa \mathrm{B}-\alpha$ degradation and NF- $\kappa \mathrm{B}$ activation ${ }^{27}$. In this study, USP15 overexpression and silencing had no effect on the expression of ІкB- $\alpha$ and p-IкB- $\alpha$ in U266 cells, and its overexpression did not affect ІкB- $\alpha$ ubiquitination, which suggest that USP15 may regulate NF$\kappa B p 65$ expression in an IкB- $\alpha$-independent manner. However, USP15 regulates IKB- $\alpha$ ubiquitination and nuclear translocation of NF- $\mathrm{kB}$ in TNF- $\alpha$-stimulated
HeLa cells ${ }^{13}$. Overall, these data suggest an important role for TNF- $\alpha$ stimulation in the regulation of USP15mediated expression and ubiquitination of IкB- $\alpha$ and NF- $\kappa B$ activity. Our data also demonstrated that PDTC treatment significantly inhibited the USP15-mediated expression of NF- $\mathrm{kB}$-regulated gene products in MM cells. Inhibition of NF-kBp65 expression in MM cells significantly reduced proliferation and apoptosis and decreased $\mathrm{Bcl}-2$ and $\mathrm{Bcl}-\mathrm{xL}$ expression ${ }^{28,29}$. NF- $\mathrm{kB}$ inactivation decreases $\mathrm{Bcl}-2, \mathrm{Bcl}-\mathrm{xL}$ and Survivin expression and subsequently activates Caspase-3, which results in MM cell apoptosis ${ }^{30,31}$. These results indicate that USP15 regulates MM tumorigenesis through activating a feedback loop with NF-kBp65.

In conclusion, our results indicate that NF-kBp65 is involved in the regulation of USP15 in MM proliferation and apoptosis and that USP15 inhibits MM apoptosis through activating a feedback loop with NF-kBp65. Together, this suggests that USP15 may be a potential therapeutic target for MM. 


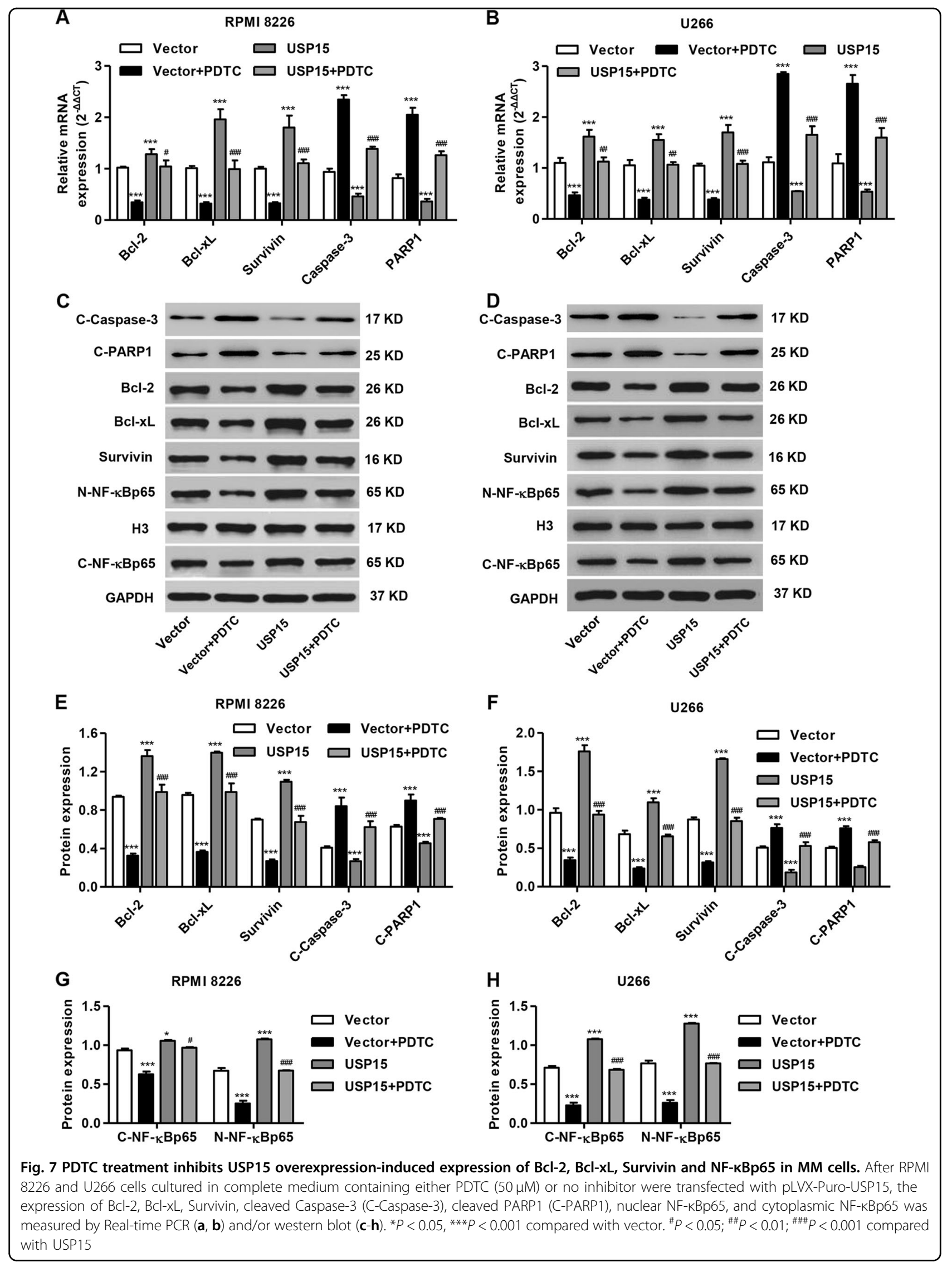




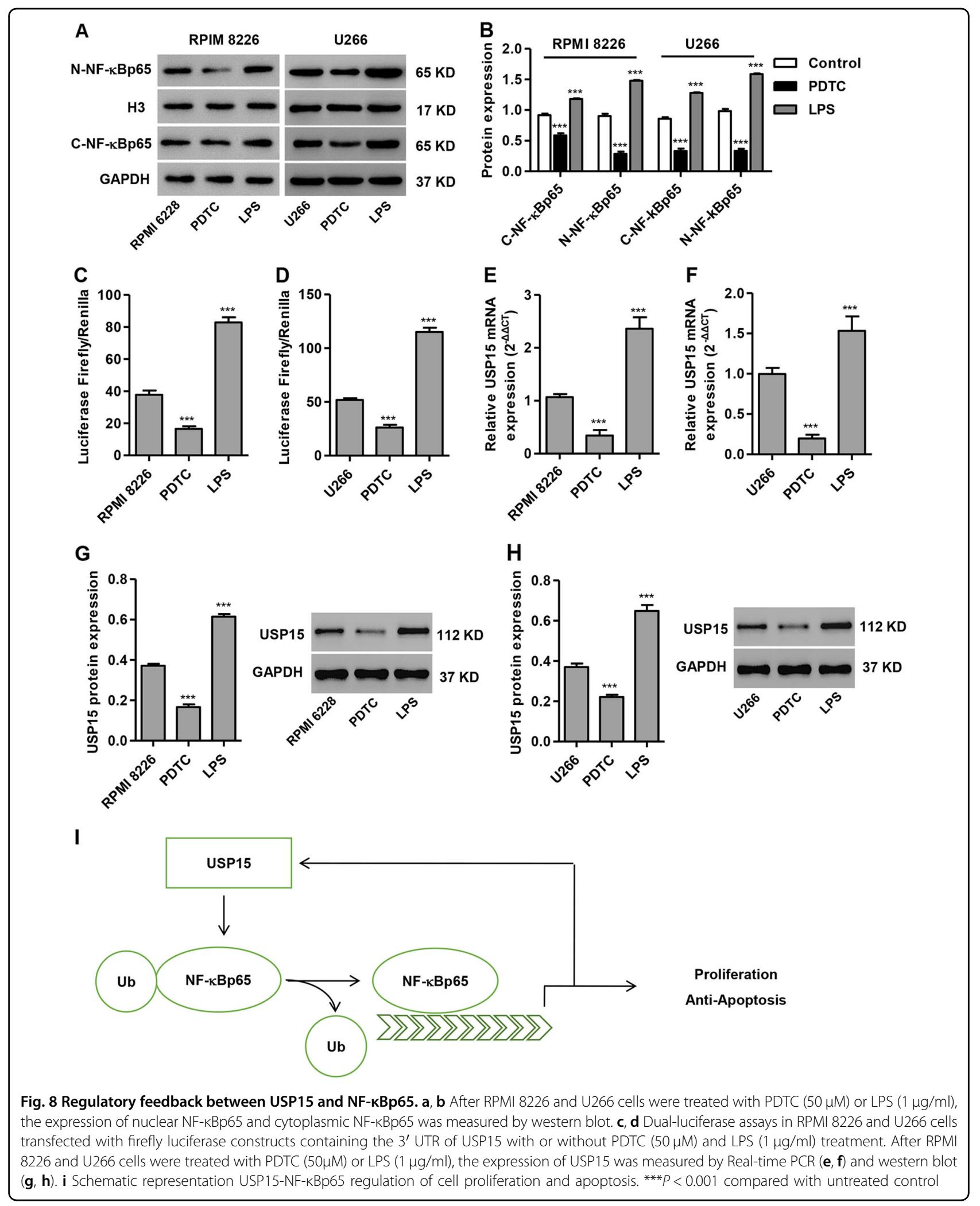




\section{Acknowledgements}

This study was supported by the Shanghai Pujiang Program (15PJ1410700) and the National Natural Science Foundation of China (81470360 and 81670193)

\section{Author details}

${ }^{1}$ Shanghai Jiahui International Hospital Cancer Center, Shanghai 200233, China ${ }^{2}$ Department of Hematology, Shanghai Changzheng Hospital, Second Military Medical University, Shanghai 200433, China. ${ }^{3}$ Department of Hematology, Renji Hospital affiliated to Shanghai Jiaotong University School of Medicine, Shanghai 200127, China

\section{Conflict of interest}

The authors declare that they have no conflict of interest.

\section{Publisher's note}

Springer Nature remains neutral with regard to jurisdictional claims in published maps and institutional affiliations.

Received: 16 May 2018 Revised: 21 August 2018 Accepted: 4 September 2018.

Published online: 20 November 2018

\section{References}

1. Viziteu, E. et al. RECQ1 helicase is involved in replication stress survival and drug resistance in multiple myeloma. Leukemia 31, 2104-2113 (2017).

2. Fakhri, B., Fiala, M. A., Tuchman, S. A. \& Wildes, T. M. Undertreatment of older patients with newly diagnosed multiple myeloma in the era of novel therapies. Clin. Lymphoma Myeloma Leuk. 18, 219-224 (2018).

3. Kazandjian, D. Multiple myeloma epidemiology and survival: a unique malignancy. Semin. Oncol. 43, 676-681 (2016).

4. Fulciniti, M. et al. Functional role and therapeutic targeting of p21-activated kinase 4 in multiple myeloma. Blood 129, 2233-2245 (2017).

5. Neemat, K., Rania, K. Tarek, M. \& Hamdy, A. A. Effect of 13q deletion on IL-6 production in patients with multiple myeloma: a hypothesis may hold true. Clin. Lab. 60, 1393-1399 (2014).

6. lida, S. et al. Bortezomib plus dexamethasone versus thalidomide plus dexamethasone for relapsed or refractory multiple myeloma. Cancer Sci. 109, 1552-1561 (2018)

7. Pulte, D., Gondos, A. \& Brenner, H. Improvement in survival of older adults with multiple myeloma: results of an updated period analysis of SEER data. Oncologist 16, 1600-1603 (2011).

8. Chen, Z. et al. MicroRNA-300 regulates the ubiquitination of PTEN through the CRL4B(DCAF13) E3 ligase in osteosarcoma cells. Mol. Ther. Nucleic Acids 10, 254-268 (2018)

9. Wang, M. et al. SYNJ2BP promotes the degradation of PTEN through the lysosome-pathway and enhances breast tumor metastasis via PI3K/AKT/ SNAl1 signaling. Oncotarget 8, 89692-89706 (2017).

10. Torre, S. \& Polyak, M. J. USP15 regulates type I interferon response and is required for pathogenesis of neuroinflammation. Nat. Immunol. 18, 54-63 (2017).

11. Padmanabhan, A., Candelaria, N. \& Wong, K. K. USP15-dependent lysosomal pathway controls p53-R175H turnover in ovarian cancer cells. Nat. Commun. 9, 1270 (2018).
12. Liu, W. T. et al. TGF-beta upregulates the translation of USP15 via the PI3K/AKT pathway to promote p53 stability. Oncogene $\mathbf{3 6}, \mathbf{2 7 1 5 - 2 7 2 3}$ (2017).

13. Schweitzer, K., Bozko, P. M., Dubiel, W. \& Naumann, M. CSN controls NFkappaB by deubiquitinylation of IkappaBalpha. EMBO J. 26, 1532-1541 (2007).

14. Borner, C. \& Kraus, J. Inhibition of NF-kappaB by opioids in T cells. J. Immunol. 191, 4640-4647 (2013).

15. Kim, J. et al. Regulation of STAT3 and NF-kappaB activations by S-nitrosylation in multiple myeloma. Free Radic. Biol. Med. 106, 245-253 (2017).

16. Milano, A., Perri, F. \& Caponigro, F. The ubiquitin-proteasome system as a molecular target in solid tumors: an update on bortezomib. Onco. Targets Ther. 2. 171-178 (2009)

17. Meshram, S. N. et al. FBXO32 activates NF-kappaB through IkappaBalpha degradation in inflammatory and genotoxic stress. Int. J. Biochem. Cell Biol. 92, 134-140 (2017).

18. Hosono, M. et al. Interaction of $\mathrm{Ca}(2+)$-dependent activator protein for secretion 1 (CAPS1) with septin family proteins in mouse brain. Neurosci. Lett. 617, 232-235 (2016).

19. Bar-Natan, M. et al. Bone marrow stroma protects myeloma cells from cytotoxic damage via induction of the oncoprotein MUC1. Br. J. Haematol. 176 929-938 (2017).

20. Zheng, Z. et al. Inhibition of thioredoxin activates mitophagy and overcomes adaptive bortezomib resistance in multiple myeloma. J. Hematol. Oncol. 11, 29 (2018).

21. Guo, W. et al. Up-regulated deubiquitinase USP4 plays an oncogenic role in melanoma. J. Cell Mol. Med. 22, 2944-2954 (2018).

22. Peng, L., Hu, Y., Chen, D., Jiao, S. \& Sun, S. Ubiquitin specific peptidase 21 regulates interleukin-8 expression, stem-cell like property of human renal cell carcinoma. Oncotarget 7, 42007-42016 (2016).

23. Chen, $X$. et al. Ubiquitin-specific protease 14 regulates cell proliferation and apoptosis in oral squamous cell carcinoma. Int. J. Biochem. Cell Biol. 79, 350-359 (2016).

24. Zou, Q. et al. USP15 stabilizes MDM2 to mediate cancer-cell survival and inhibit antitumor T cell responses. Nat. Immunol. 15, 562-570 (2014).

25. Eichhorn, P. J. et al. USP15 stabilizes TGF-beta receptor I and promotes oncogenesis through the activation of TGF-beta signaling in glioblastoma. Nat. Med. 18, 429-435 (2012).

26. Mialki, R. K., Zhao, J., Wei, J., Mallampalli, D. F. \& Zhao, Y. Overexpression of USP14 protease reduces I-kappaB protein levels and increases cytokine release in lung epithelial cells. J. Biol. Chem. 288, 15437-15441 (2013).

27. Xiao, N. et al. Ubiquitin-specific protease 4 (USP4) targets TRAF2 and TRAF6 for deubiquitination and inhibits TNFalpha-induced cancer cell migration. Biochem. J. 441, 979-986 (2012).

28. Liu, S., Zheng, L. L., Zhu, Y. M. \& Shen, H. J. Knockdown of REGgamma inhibits the proliferation and migration and promotes the apoptosis of multiple myeloma cells by downregulating NF-kappaB signal pathway. Hematology $\mathbf{2 3}$, 277-283 (2018).

29. Sun, C. et al. Resveratrol downregulates the constitutional activation of nuclear factor-kappaB in multiple myeloma cells, leading to suppression of proliferation and invasion, arrest of cell cycle, and induction of apoptosis. Cancer Genet. Cytogenet. 165, 9-19 (2006).

30. Chen, G. et al. An anti-leishmanial thiadiazine agent induces multiple myeloma cell apoptosis by suppressing the nuclear factor kappaB signalling pathway. Br. J. Cancer 110, 63-70 (2014).

31. Takeda, T. et al. Mangiferin enhances the sensitivity of human multiple myeloma cells to anticancer drugs through suppression of the nuclear factor kappaB pathway. Int. J. Oncol. 48, 2704-2712 (2016). 